\title{
15. "Hello computer, how am I feeling?", Case Studies of Neural Technology to Measure Emotions
}

\author{
${ }^{1}$ Ian Daly and ${ }^{2}$ Duncan Williams \\ ${ }^{1}$ Brain-Computer Interfacing and Neural Engineering Lab, Department of Computer Science \\ and Electronic Engineering, University of Essex, Colchester, UK, CO4 3SQ \\ ${ }^{2}$ Digital Creativity Labs (Computer Science department), University of York, York, UK, YO1O \\ $5 D D$
}

\section{Contents}

1. Introduction

2. Neural technology-based measures of emotions

3. Case study 1: Affective brain-computer music interfacing

3.1 Introduction

3.2 Outline

3.3 Affective state detection

3.4 Evaluation

3.5 Results

3.6 Discussion

4 Case study 2: Driving

4.1 Background

4.2 Method

4.3 Data collected

4.4 Analysis techniques

4.5 Results

4.6 Conclusions

5 Conclusions and future research directions

References

\begin{abstract}
Emotion is a core part of the human experience. Many artistic and creative applications attempt to produce particular emotional experiences, for example films, games, music, dance, and other visual arts. However, while emotional states are ubiquitous, they are also complex, proving difficult to describe to others by conventional psychometric means (e.g., traditional selfreport mechanisms).

Neural technology offers the potential to circumvent these difficulties by allowing the creation of a real-time, objective, metric of felt emotion to assist in emotionally-driven experience design across a range of disciplines.

This chapter discusses how neural technology based on the processing of the electroencephalogram may be used to measure human emotions in natural environments. We also present a set of case studies of applications which use neural technology to measure emotions. We are particularly interested in the use of neural technology to inform applications
\end{abstract}


which can respond to the felt-experience of the individual. We describe two case studies focused on driving scenarios and brain-computer music interfacing.

The chapter concludes with a discussion of the challenges inherent in developing neural technology to measure emotion and a set of suggestions for future research directions in developing applications that use neural technology as an objective measure of emotion.

\section{Introduction}

The experience of emotion is a core part of our humanity, it is the driver behind much of our behavior, and forms the bedrock of the motivations behind many of our actions throughout our lives. Yet, despite the overwhelming importance of emotion in defining who we are and what we do, we still do not fully understand the neural or physiological bases of emotion (Darwin, 1998; Reeve, 2014).

An emotion may, broadly speaking, be defined as an action, or a pattern of multiple actions that occur in response to one or more external stimuli or that arise spontaneously in an individual's (Barrett, Mesquita, Ochsner, \& Gross, 2007). Emotions may arise either as a result of environmental stimuli or from the body (for example by memories or in response to physical pain or discomfort) (Mier et al., 2010).

In order to scientifically understand emotions, it is first necessary to develop a quantifiable framework by which emotion may be measured. Efforts to do so in the field of Psychology have resulted in the development of multi-dimensional frameworks for describing emotions within a variety of contexts as well as quantifiable labelling systems to allow the systematic description of emotions.

The scientific study of emotion first seeks to identify a system by which different emotions (affective states) may be categorized. A large number of different systems have been proposed to achieve this goal, including category labelling systems and multi-dimensional systems spanning different numbers of dimensions.

The multi-dimensional schemes developed to categorize emotions include the valence-arousal circumplex model of affect (Russell, 1980), the three dimensional Schimmack and Grob model (Schimmack \& Grob, 2000), and other multidimensional models such as the three dimensional pleasure, arousal, dominance model (Mehrabian, 1996). Alternatively category labelling systems, such as the Geneva emotional music scale (GEMS) (Zentner, Grandjean, \& Scherer, 2008) or the Ortony, Clore, and Collins category model (Ortony, Clore, \& Collins, 1988) may be used to categorize affect via a set of discrete labels.

Multidimensional categorization schemes map affective states to a continuous space. For example, the valence-arousal circumplex model maps emotions onto a continuous twodimensional plane and specific affective states can be placed at any location. This approach has the advantage of allowing fine grained measurement and differentiation of affective states, but may not be the most suitable or accurate categorization. 
Indeed it has been argued that, despite the wide-spread use of the two dimensional valencearousal circumplex, emotions are not accurately represented using just two dimensions and dimensionless category labelling schemes, such as GEMS, are more accurate (Fontaine, Scherer, Roesch, \& Ellsworth, 2007).

Arguably, the most widely used emotion categorization system is the two dimensional valencearousal circumplex (Russell, 1980). This framework classifies all emotions along two axes, valence (the pleasantness or unpleasantness of the emotion) and arousal (the level of excitement of the emotion). Thus, for example, "fear" may be placed on the two dimensional plane of the circumplex at a position with low (negative, or 'unpleasant') valence and high (positive, or 'exciting') arousal, while "excitement" may be placed on the circumplex at a position of high valence (very pleasant) and high arousal (very exciting).

The valence-arousal circumplex is illustrated in Figure 1.

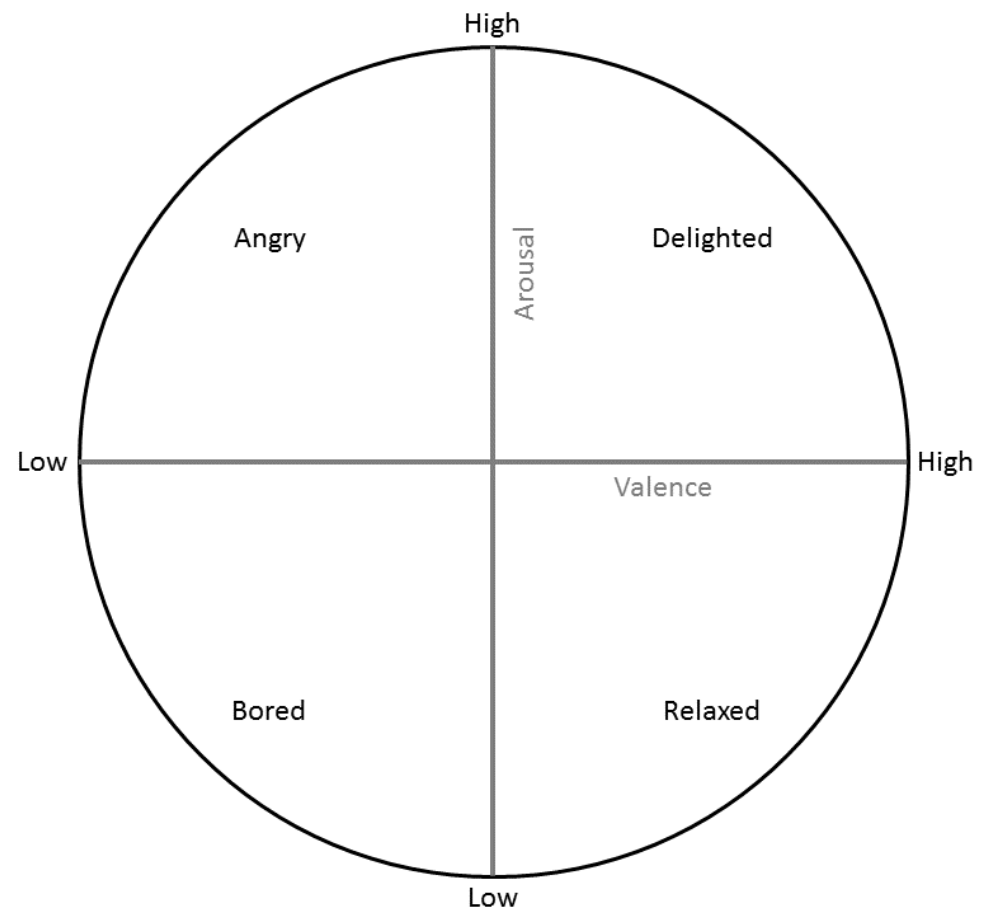

Figure 1. The valence-arousal circumplex.

This two dimensional framework has been extended by others to include a third dimension, tension, which is defined as the level of strain or stress a particular emotion involves (Schimmack \& Grob, 2000). Indeed, some research suggests this three dimensional framework may provide a more complete and accurate description of emotional responses (Ian Daly et al., 2014). However, others suggest that the linear two or three dimensional relationships suggested by these frameworks is misleading and in fact emotion is a highly non-linear, multi-dimensional experience that is not well described by two or three dimensional Cartesian geometry (Fontaine et al., 2007). 
Efforts to quantify and describe emotions in a multi-dimensional space have made use of labels to describe emotional responses in a discipline specific framework. For example, the Geneva music scale (GEMS) attempts to describe emotional responses to a piece of music via a set of selected adjectives, which an individual is asked to select from in order to describe their current emotional experience. However, such systems are not as readily amenable to quantification or translation between languages or cultures. Thus, for the present, the two- or three-dimensional quantifiable frameworks, such as the valence-arousal circumplex, remain widely used.

Although emotions are ubiquitous in life and analogous to vision or hearing in their effect on our moment-by-moment perception of the world, they, historically, have received far less interest from the scientific community (Kassam, Markey, Cherkassky, Loewenstein, \& Just, 2013). Consequently, the neural basis of emotions is far less well-understood than the neural basis of our other senses (Kassam et al., 2013).

Meta-analysis of neuroimaging studies investigating the neural basis of emotion has revealed a network of brain regions that are involved in emotional responses (Koelsch, 2010). This network includes, amongst other brain areas, the amygdala, hippocampal formation, right ventral striatum, pre-supplementary motor cortex, left caudate nucleus, and the cingulate cortex (Koelsch, 2014).

Exploration of this network via techniques such as functional magnetic resonance imaging (fMRI) (which measures slow changes in concentrations of oxy-hemoglobin throughout the brain) or electroencephalography (EEG) (which measures fast changes in electro-potential in the cortex) has revealed particular patterns of neural activity to relate to specific felt emotions. For example, changes in a participants felt valence (how pleasant or unpleasant they find a stimulus or situation), also referred to as 'approach-withdrawal' behavior (Hunter \& Schellenberg, 2010), involves changes in activity in the paralimbic system and amygdala, amongst others (Blood \& Zatorre, 2001). These changes in neural activity are also reflected in the EEG, for example in the prefrontal asymmetry (Coan \& Allen, 2004a).

However, there remains much to discover about how our brains, and our bodies, work together to generate the experience of emotions.

Better understanding of the basis of emotions can lead to numerous benefits. The most valuable of these, arguably, relate to medical interventions for individuals living with mood disorders such as depression or anxiety (Hou et al., 2017). However, more accurate understanding of the basis of emotions can also lead to numerous other applications, including within the field of neuroergonomics to design systems that better respond to our current felt emotions in a way that is useful or beneficial to us.

Accurately identifying an individual's current emotion is not trivial. One could simply ask an individual, or a group of individuals, what emotions they are feeling after exposure to a stimulus. However, individuals respond to a stimulus in a way that depends on their lived experiences, memories, current mood, recent levels or rest, and other emotional and physiological wants and desires (which themselves change from moment to moment) (Barrett et al., 2007). Consequently, 
the way an individual emotionally responds to a situation is specific to them and may vary considerably from the way another individual may respond to the same situation.

Furthermore, individuals may also not be able to accurately evaluate or describe their own emotions. The particular choice of words that an individual uses to describe their current emotions are largely dependent on their background and can change radically, even between individuals with very similar backgrounds (e.g. between siblings). One could use a standard scale to allow individuals to report their emotions (for example, by providing a discrete set of prechosen key-words as used in the Geneva emotional music scales system (Vuoskoski \& Eerola, 2011)), but even this approach is not without flaws as each individual may interpret the chosen words or scale differently.

Finally, individuals may not be honest when reporting some of their emotional responses. This is particularly the case with emotions at the more extreme ends of the scale or in response to stimuli which may have some associated socially-dictated negative or embarrassing connotations for an individual.

These problems are increasingly being tackled by making use of our growing understanding of the neural basis of emotions. Specifically, neural technology is being developed and used to produce systems that are able to measure and respond to an individual's current emotional (affective) state.

Neural technology allows for the development of a set of metrics that are able to provide objective measures of an individual's current felt-emotions. Such metrics have numerous advantages. For example, they can allow objective measurement of an individual's felt response to a stimuli or situation. This has numerous applications, including in Psychology or Neuroscience experiments to overcome the problems with traditional emotion assessment methods outlined above.

Neural technology-based affect metrics may also be used within a neuroergonmic context to allow a system to respond to neural measures of an individual's current felt affective state. For example, an application could identify when a user is becoming frustrated with some aspect of the application and adjust accordingly.

Recent research on the development of neural technology-based metrics for measuring emotions makes use of measures of an individual's physiological state. Some initial applications have been developed that make use of these metrics for applications designed to aid people in their interactions with the world.

Of course, the use of neural technology to measure physiological correlates of emotion introduces its own specific considerations. For example, physiological correlates of emotion differ between individuals as a result of inter-person differences in physiology, signal noise, and differences in measured signal strength. These factors mean identifying a common signal component that can be used as a reliable indicator of emotion presents a considerable challenge. 
In this chapter we first introduce how neural technology, and in particular neural technology based on the EEG, can be used to provide a metric of an individual's affective state before introducing some existing neural technology-based methods that have been developed to monitor affective states. We then go on to describe two case studies that illustrate how these metrics may be used to monitor affect and the applications that may be built around these metrics. Finally, we discuss some possible future directions in the development of this technology and the associated challenges that still need to be overcome.

\section{Neural technology-based measures of emotions}

There are several neuro-imaging technologies available to measure activity in the brain and these vary in resolution (in space, time, and frequency), as well as in their cost and portability. Many of these technologies can be used to provide an objective measure of felt emotion (Canli, Desmond, Zhao, Glover, \& Gabrieli, 1998; Ishii et al., 2014; Skouras, Gray, Critchley, \& Koelsch, 2013). However, of the available neuroimaging technologies the electroencephalogram (EEG) is generally considered to be the most suitable for providing real-time, portable, and accurate measures of an individual's current affective state due to its relative low cost, portability, high temporal and spectral resolutions, and its ability to be used directly without invasive surgery (Niedermeyer \& Silva, 2005).

EEG is a measurement of summed electrophysiological activity from large numbers of cortical neurons. It is recorded from the surface of the scalp by either passive or active electrodes and has a time resolution in the order of hundreds or thousands of samples per second (Niedermeyer \& Silva, 2005).

When using the EEG to measure an individual's affective state there are a few key features that have been reliably identified and shown to allow accurate real-time measurement and tracking of an individual's current affective state. The most widely used of these metrics are:

1. The pre-frontal asymmetry

2. Midline theta bandpower

3. Functional connectivity

Prefrontal EEG asymmetry is a measure of the relative difference in EEG activity in the left and right hand prefrontal cortices (Coan \& Allen, 2004b). The ratio of EEG activity in this region is known to vary within specific frequency bands - including, but not limited to, the alpha (8$13 \mathrm{~Hz})$, beta $(13-25 \mathrm{~Hz})$, and theta $(4-8 \mathrm{~Hz})$ frequency bands - as a function of approachwithdrawal behavior in response to numerous different types of stimuli, including images (Irene Winkler, Mark Jäger, Vojkan Mihajlović, 2010), videos (Jones \& Fox, 1992), and music (Schmidt \& Trainor, 2001). This is expressed in the "hemispheric valence hypothesis", which states emotions related to approach to a stimulus (i.e. positive responses to a stimulus) are processed more in the left prefrontal cortex, while withdrawal (negative) responses to a stimulus are processed more in the right prefrontal cortex (Canli et al., 1998; Silberman \& Weingartner, 1986). Additionally, the parietotemporal cortex in the right hemisphere has been shown to be involved in autonomic behavior, while arousal is also involved in suppression of alpha power in 
the right posterior cortex and increases in theta EEG power in the left prefrontal lobe (Rogenmoser, Zollinger, Elmer, \& Jäncke, 2016).

A less frequently explored, yet robust, metric of felt affect is the midline theta, the change in power of the EEG within the theta frequency band as measured along the central sulcus (the midline) (Aftanas, Varlamov, Pavlov, Makhnev, \& Reva, 2001). This metric has been reported to be positively correlated with the valence of an affect (Sammler, Grigutsch, Fritz, \& Koelsch, 2007). Specifically, the more pleasant a stimulus or situation is felt to be by the individual the greater their midline theta activity.

Finally, functional connectivity (a measure of the statistical relationships between different regions of the brain (Sporns, 2007)) has also been reported to be used as a metric of the felt emotional response to a stimuli (Ian Daly et al., 2014). Functional connectivity refers to the statistical relationships between neural activity signals recorded in different locations within, or on the surface of, the brain (Raichle, 2011). Larger statistical relationships between different signals suggests a meaningful relationship between the measured regions of the brain.

Functional connectivity may be differentiated from anatomical connectivity, which measures anatomical connections between different brain regions (Sporns, 2007). For example, in the case of functional connectivity a statistical relationship may exist as a result of a common anatomical connection both measured brain regions share with a third, unmeasured, brain region (Sporns, 2007).

Functional connectivity has been shown to change a result of changes in felt affect in a growing number of studies, including in responses to music (Nicolaou et al., 2017) and after watching film clips chosen to explicitly specific emotional response (Lee \& Hsieh, 2014). Furthermore, functional connectivity patterns have also been observed to vary significantly as a result of major depression (Fingelkurts et al., 2007) and other disorders of mood (Davidson, Abercrombie, Nitschke, \& Putnam, 1999) within clinical populations.

Human emotion is increasingly understood to be an embodied experience (Effron, Niedenthal, Gil, \& Droit-Volet, 2006). Felt emotional response to a stimuli or situation is not just a product of the activity in the brain but also a product of the autonomic response system (Kreibig, 2010). Thus, accurate physiological metrics of affective states may be identified by incorporating other physiological measures recorded from the body. These may include measures such as the heart rate, galvanic skin response, respiration rate, and blood oxygenation levels (Kreibig, 2010).

The relationships between physiological responses to emotion and specific categorization systems for emotion are varied and complex. For example, the heart rate (as measures by the ECG) is well known to correlate with felt arousal, but also relates to felt valence responses to some types of stimuli, such as music (I. Daly et al., 2015).

A system which combines some or all of these physiological measures along with neural measures of EEG indices of affect is defined as a 'hybrid measure' and is increasingly used in, amongst other applications, hybrid brain-computer interface systems for monitoring and measuring affective states in individuals by recording and classifying combinations of neural and 
physiological activity (Fazli et al., 2012; Müller-Putz et al., 2011; Nicolas-Alonso \& Gomez-Gil, 2012; Pfurtscheller et al., 2010).

It may be argued that measures of the autonomic systems alone may be sufficient to accurately measure affective responses to stimuli and that, in fact, there is no need for measures of the neural response to stimuli. However, this has been shown not to be the case (Ian Daly et al., 2015). Indeed hybrid measures incorporating both neural and physiological measures of affective states have been shown to be able to more accurately measure an individual's current affective state than either set of measures alone (Ian Daly et al., 2015).

We conclude that there are a growing range of neural technology-based measures of emotions. These may be used in a wide variety of applications, as we will illustrate in our case study below.

\section{Case study 1: Affective brain-computer music interfacing}

\subsection{Introduction}

In this section we introduce and describe a case study of a brain-computer music system (BCMI) developed to monitor and respond to an individual's current affective state. This system was originally described in full in (Ian Daly et al., 2016a) and is presented here in brief.

Music is a unique and powerful method for influencing an individual's current affective state (Sacks, 2006). Even very short clips of music can produce varied and powerful emotional responses in the listener. For example, something as simple as a few piano keys played over a few seconds can produce fear, excitement, contentment, or melancholy depending on the exact nature of the music, the current mood of the listener, and other extraneous factors.

Because of this, music is increasingly being explore as a tool in therapy (Bradt, Magee, Dileo, Wheeler, \& McGilloway, 2010; Erkkilä et al., 2011; Livingstone \& Thompson, 2009). Indeed, music therapy is increasingly used as a therapeutic technique to help facilitate emotional communication in patients with a range of emotional disorders. The BCMI described in this case study is an attempt to provide a form of automated music therapy.

\subsection{Outline}

An overview of the BCMI developed and used in this case study is illustrated in Figure 1.0. 


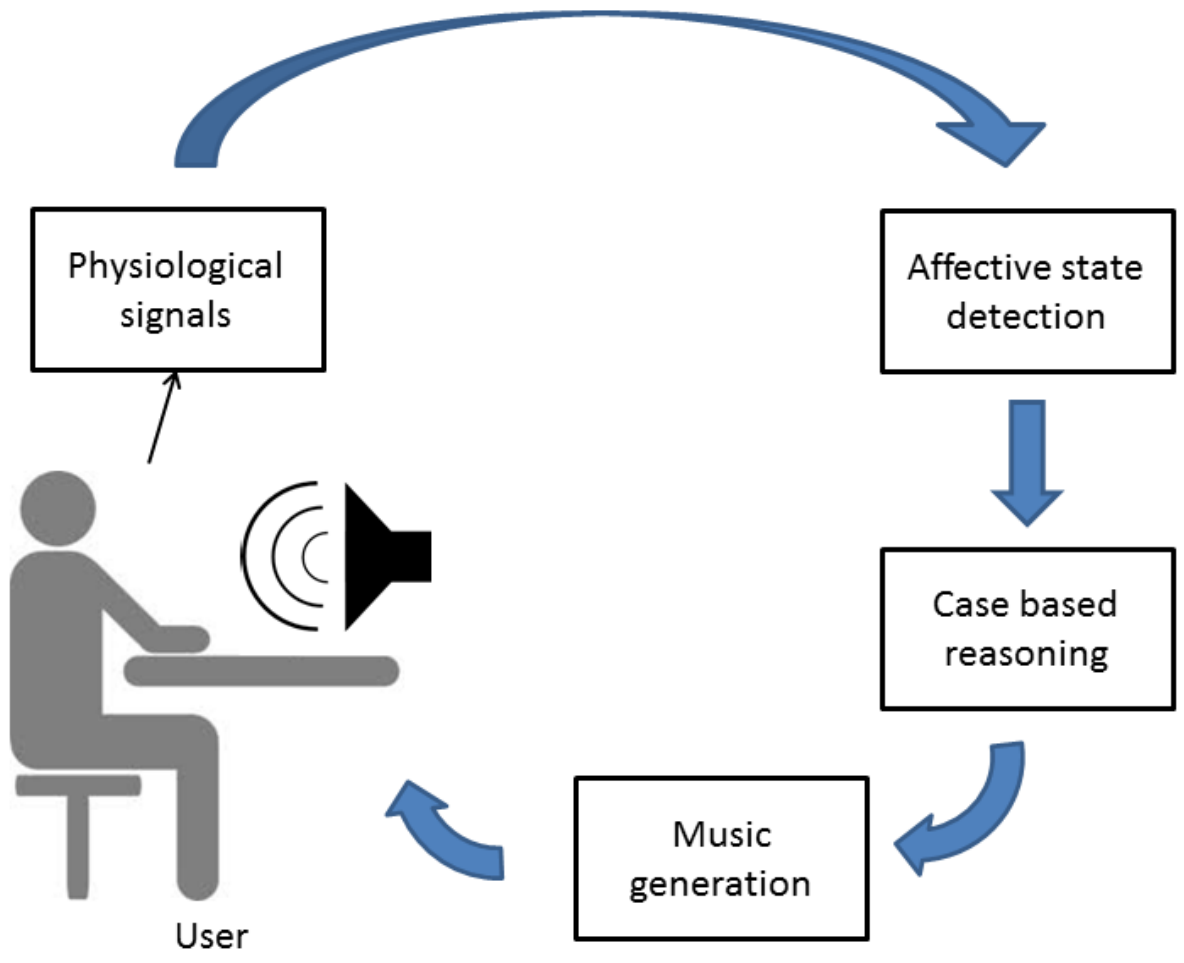

Figure 2. Schematic of the BCMI system developed in case study 1.

The following physiological signals are recorded from the users at a sample rate of $1,000 \mathrm{~Hz}$ : EEG, electrocorticogram (ECG), galvanic skin response, and respiration. These are then used to attempt to detect the users current affective state, as described in section 3.3 below. A case based reasoning system (Webber, Aha, Muñoz-Ávila, \& Breslow, 2000) is then employed to identify the ideal set of music to play to the participant in order to move them from their current affective state to a new target affective state. Finally, a music generator (Williams et al., 2015), (Williams et al., 2017) is used to generate the music identified by the case based reasoning system and play it to the BCMI user.

A typical use case envisioned for this BCMI is modulating the affective state of an individual who is feeling mildly depressed, i.e. in a low valence, low arousal state (Russell, 1980). In this case the affective state detection system would identify the user's current affective state as 'low valence', 'low arousal'. The case-based reasoning system would then identify the set of musical features that would be most effective at improving that users affective state, before the music generator is used to generate and play this music to the user.

Of note, this system does not make any a-priori assumptions about what musical transitions would be most effective for each user. This is motivated by evidence that each individual responds to music differently (Eerola, 2012). For example, a piece of music that makes one 
person happy may bore or frustrate another. Instead, the optimal set of music pieces to produce a target affect in an individual is determined in a training session with each individual prior to use of the BCMI.

\subsection{Affective state detection}

The affective state detection method used in this BCMI makes use of EEG band powers in a range of frequency and spatial regions. Specifically, the EEG is sub-divided into channel sets placed over 10 key regions of the scalp, including the prefrontal, central, and occipital regions. These candidate features were chosen based on their reported successful use in classifying affective states in prior work. For example, a system proposed in (Stikic, Johnson, Tan, \& Berka, 2014) uses these spatial regions to train a feature selector and classifier. This use of a wide range of different spatial regions to detect an individual's affective state is supported by work from several authors that suggests a broad involvement of different spatial regions in affective states (Ian Daly et al., 2014; Heller, 1993; Rogenmoser et al., 2016). The EEG within each region is also filtered into 10 different frequency bands, encompassing all the key frequency bands widely used in EEG analysis and their sub-bands (Schomer \& Lopes de Silva, 2011).

In total 10 different spatial regions and 10 different frequency bands are used in the study, resulting in 100 candidate EEG features. These were complemented by additional features describing each of the other physiological signals used. Specifically, the mean heart rate (peakto-peak intervals), mean blood oxygenation rate (peak-to-peak intervals), dominant frequency of the respiration rate, and mean amplitude of the change in galvanic skin response were also used as descriptive features. This resulted in a total of 104 candidate features.

A stepwise linear regression feature selection method was then used to identify the subset of these features that was most effective at classifying the users current affective state. Affective states were quantized into 9 different values. Each of these quantized affective states is represented by a tuple composed of valence and arousal, each of which can take one of three values: high, medium, and low.

A support vector machine (SVM) classifier was trained and used to attempt classification of the affective states. Specifically, we attempt to identify a participants reported felt affective state via an SVM classifier trained on that participants EEG and physiological features.

Full details of the development of this metric are described in (I. Daly et al., 2016) and its implementation in the BCMI is described in more detail in (Ian Daly et al., 2016b).

\subsection{Evaluation}

The BCMI was evaluated with a cohort of 22 healthy participants over a series of experimental sessions (Ian Daly et al., 2016b). The first 4 sessions were used to train the case-based reasoning system and the affective state detection method, while the final session was used to evaluate the system. 
Training and evaluation sessions took place over several, non-consecutive, days. The training sessions were used to map relationships between music features and individual affective responses in order to train the case-based reasoning system. They were also used to train the feature selection and classification steps of the affective state recognition system.

Additionally, the BCMI was also evaluated in a follow-up case study with a single individual with Huntington's disease to demonstrate its ability to also work for users with medical conditions who may stand to benefit from BCMI use (Ian Daly et al., 2017).

Huntington's disease affects 7-12 in 100,000 people. It is a progressive central nervous system disorder that, over 10-12 years, reduces movement control and impairs cognition and behavior. It also impairs an individual's emotion regulation and, it is suggested, music therapy techniques (such as BCMI), may be beneficial (Bates, Tabrizi, \& Jones, 2014; Pridmore, 1990).

Evaluation - with both the healthy user cohort and the individuals with Huntington's disease was performed by asking the system to attempt to make a series of specific modulations to the user's affective states. Four objectives were selected, based upon potential music therapy use case scenarios. These objectives were:

1. Make the user happy (move the user from a low valence to a high valence state).

2. Calm the user (reduce the user's arousal).

3. Reduce stress (increase valence, while simultaneously reducing arousal).

4. Excite the user (increase the users felt arousal).

In each trial a target change in affective state was selected at random and participants were not informed about which affective state transition was being targeted. The music was then generated according to the rules of the case-based reasoning system determined in the training sessions of the experiment.

The success of the BCMI at each of these objectives was measured by comparing the resulting trajectories of changes in user-reported affective states with trajectories that would be achieved by a random system, i.e. a system that just played randomly selected music to the users.

Users reported their current felt affect throughout the experiment using the real-time affect reporting tool FEELTRACE, which allows users to report, in real-time, their current felt affective states as a coordinate trajectory on the 2-dimensional valence-arousal circumplex as they listen to the music (Cowie et al., 2000). We hypothesized that if the BCMI was not working, users affective state trajectories, as reported via FEELTRACE, would be random. On the other hand, if the BCMI was working correctly, changes in valence and arousal that correspond to the target objectives would be observed. Full details of the experiment design are described in (Ian Daly et al., 2016b).

\subsection{Results}

The affective state detection method developed in the BCMI was able to identify affective states with statistically significant classification accuracies for the majority of participants. This is best 
illustrated in the work described in (I. Daly et al., 2015), which evaluates the affective state detection method in isolation with a separate population of healthy participants.

The results are listed in Table 1 for valence classification and in Table 2 for arousal classification.

Table 1. Classification accuracies resulting from our affective state detection method for differentiating high and low valence conditions. Statistical significance $(p)$ is estimated from the binomial distribution. 'Acc' denotes accuracy and 'STD' denotes standard deviation.

\begin{tabular}{|l|l|l|l|}
\hline Participant & Acc. (mean) & Acc. (STD) & P \\
\hline 1 & 0.753 & 0.066 & $<0.001$ \\
\hline 2 & 0.597 & 0.059 & 0.032 \\
\hline 3 & 0.619 & 0.084 & 0.019 \\
\hline 4 & 0.609 & 0.087 & 0.055 \\
\hline 5 & 0.628 & 0.069 & 0.014 \\
\hline 6 & 0.633 & 0.079 & 0.034 \\
\hline 7 & 0.629 & 0.081 & 0.034 \\
\hline 8 & 0.599 & 0.057 & 0.018 \\
\hline 9 & 0.671 & 0.063 & 0.009 \\
\hline 10 & 0.537 & 0.122 & 0.160 \\
\hline 11 & 0.629 & 0.048 & 0.006 \\
\hline 12 & 0.649 & 0.054 & 0.002 \\
\hline 13 & 0.647 & 0.067 & 0.006 \\
\hline 14 & 0.705 & 0.072 & $<0.001$ \\
\hline 15 & 0.549 & 0.068 & 0.067 \\
\hline 16 & 0.604 & 0.069 & 0.021 \\
\hline 17 & 0.568 & 0.059 & 0.053 \\
\hline Avg. & 0.624 & 0.071 & - \\
\hline
\end{tabular}

Table 2. Classification accuracies resulting from our affective state detection method for differentiating high and low arousal conditions. Statistical significance $(p)$ is estimated from the binomial distribution. 'Acc.' denotes classification accuracy and 'STD' denotes standard deviation.

\begin{tabular}{|l|l|l|l|}
\hline Participant & Acc. (mean) & Acc. (STD) & P \\
\hline 1 & 0.724 & 0.048 & $<0.001$ \\
\hline 2 & 0.726 & 0.074 & $<0.001$ \\
\hline 3 & 0.694 & 0.065 & $<0.001$ \\
\hline 4 & 0.779 & 0.061 & $<0.001$ \\
\hline 5 & 0.746 & 0.061 & $<0.001$ \\
\hline 6 & 0.561 & 0.060 & 0.061 \\
\hline 7 & 0.591 & 0.099 & 0.081 \\
\hline 8 & 0.701 & 0.050 & $<0.001$ \\
\hline 9 & 0.795 & 0.056 & $<0.001$ \\
\hline 10 & 0.598 & 0.099 & 0.097 \\
\hline 11 & 0.700 & 0.051 & $<0.001$ \\
\hline 12 & 0.659 & 0.053 & $<0.001$ \\
\hline
\end{tabular}




\begin{tabular}{|l|l|l|l|}
\hline 13 & 0.677 & 0.059 & 0.002 \\
\hline 14 & 0.733 & 0.057 & $<0.001$ \\
\hline 15 & 0.694 & 0.052 & $<0.001$ \\
\hline 16 & 0.729 & 0.055 & $<0.001$ \\
\hline 17 & 0.688 & 0.144 & 0.003 \\
\hline Avg. & 0.694 & 0.067 & - \\
\hline
\end{tabular}

It may be observed that for the majority of the affective state detection attempts the affective state detection method was able to identify the correct affective states with statistically significant accuracies $(\mathrm{p}<0.05)$. This demonstrates how neural engineering may be employed to accurately identify an individual's current emotions in the context of a BCMI system.

The BCMI system was able to significantly modulate the user's affective states under all scenarios in the majority of cases. Examples of the affective trajectories achieved by the system are illustrated in Figure 3. Valence and arousal are plotted as separate trajectories to allow easier interpretation of the results.
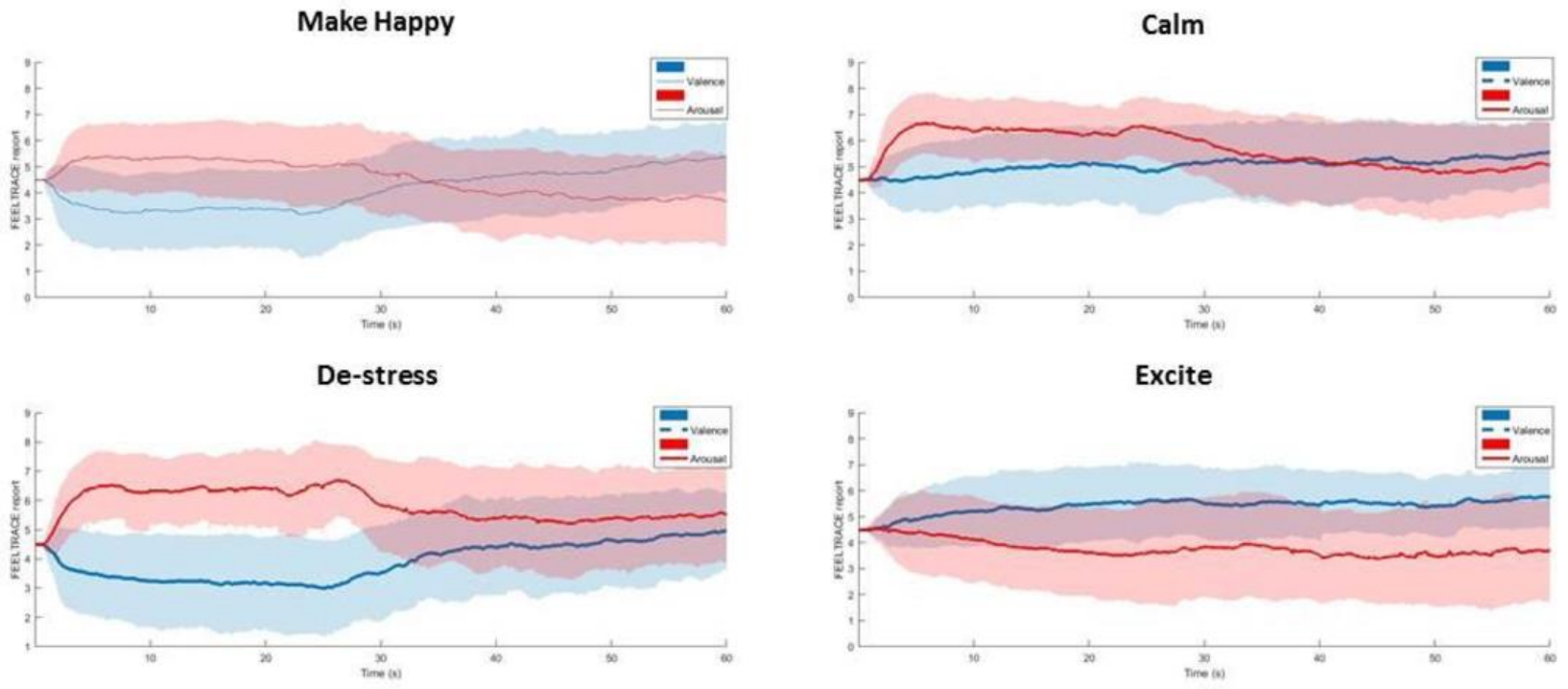

Figure 3. Examples of affective state trajectories achieved by the BCMI system. FEELTRACE report indicates the output of the FEELTRACE interface used to allow participants to report their current affective states.

It may be observed that, in each case, the BCMI was able to move the users from their starting affective state (the first 20s of the trial) to the target affective state (the last $20 \mathrm{~s}$ of the trial). The BCMI begins to attempt to change each users affective state from $20 \mathrm{~s}$ into each trial and this can be clearly seen in the plots of the mean affective state trajectories across all participants.

Specifically, there is a clear, visible change in reported affective state as the BCMI is used. For example, the third objective is to reduce the participant's level of stress ('de-stress' in the figure), which corresponds to increasing the felt valence and reducing the felt arousal (i.e. making the participant happier and calmer). This result may clearly be seen in the figure; the mean valence increases, and the mean arousal decreases. These changes in affective state are statistically significantly different from random affective state changes $(\mathrm{p}<0.05)$ as assessed by comparing to distributions of random trajectories generated under the null hypothesis. 
When the BCMI is employed with an individual with Huntington's disease in our follow-up case study, significant changes in valence are achieved with results comparable to those delivered with healthy participants. However, no significant changes in arousal are achieved. These results are illustrated in Figure 4, which illustrates the change in FEELTRACE trajectories reported by our participant for each of the BCMI evaluation goals.

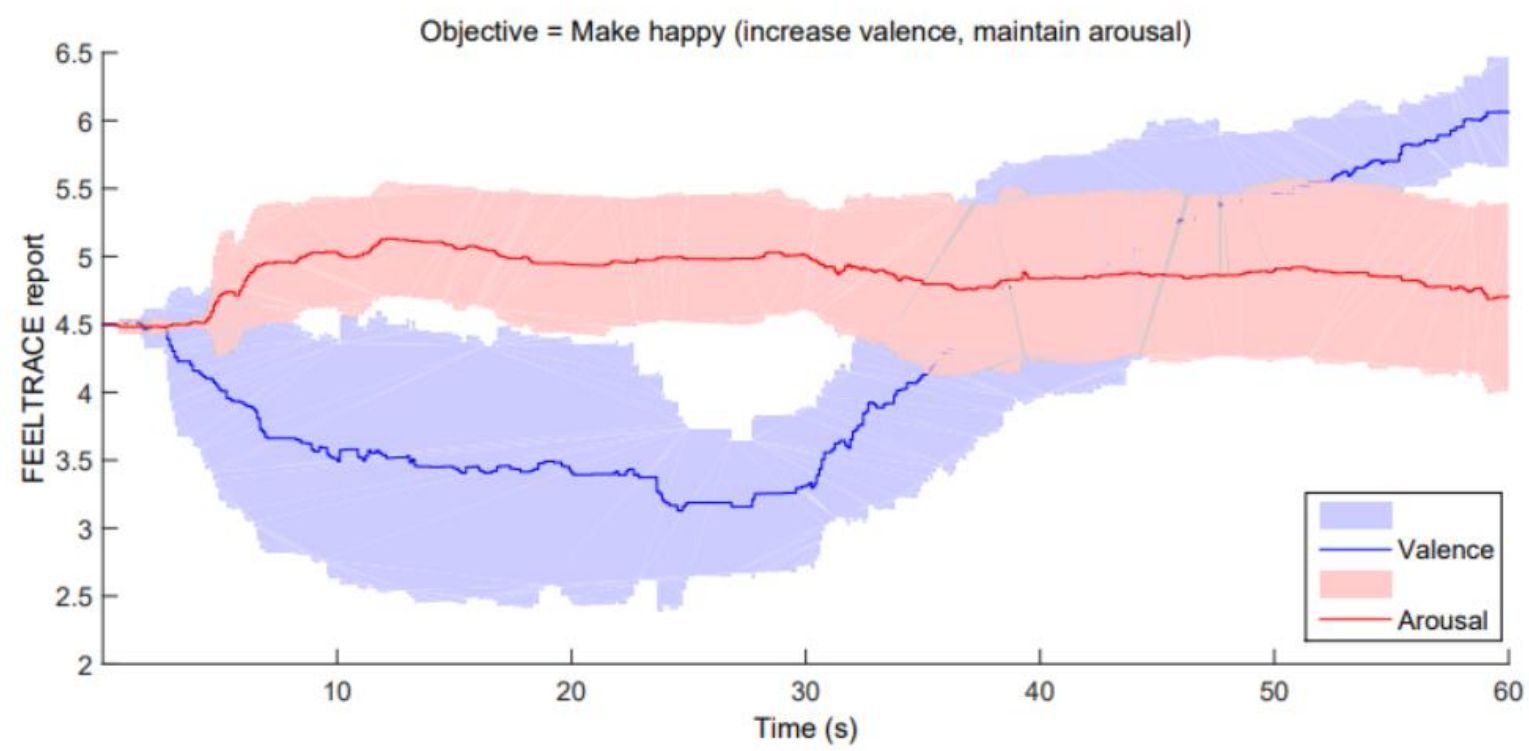

Figure 4. Affective state trajectories achieved by the BCMI system when it was employed with an individual with Huntington's disease to attempt to increase the individual's valence. FEELTRACE report indicates the output of the FEELTRACE interface used to allow participants to report their current affective states.

A full description of the results of the evaluation of the BCMI with the individual with Huntington's disease is available here (Ian Daly et al., 2017).

\subsection{Discussion}

This case study provides an example of one of the first efforts to develop an automated BCMI system for aiding music therapy. The system uses a real-time hybrid metric of affective states to monitor an individual's emotions and respond to them. The metric uses a combination of EEG and other physiological processes and is able to accurately identify and respond to a user's current affective state.

There are some considerations to be made when evaluating the results of this system. First, there is considerable variability in the reported affective states. This is likely to be a result of the large amount of known inter-person variability in individual affective responses to stimuli and large inter-person differences in neural and physiological signal properties, upon which the affective state detection method that underpins the BCMI is based.

Additionally, it may be argued that the use of the FEELTRACE interface itself is interfering with individual felt affective states. FEELTRACE is controlled by a joystick or mouse and the act of concentrating on the control of this interface may affect an individual's affective state. However, 
as FEELTRACE is used throughout all parts of the experiment its effect on felt affective states may be treated as a continuous background noise signal and is unlikely to adversely affect the classification performance of the system.

This case study provides an illustration of how neural and physiological metrics may be developed and employed to allow computer systems to monitor and respond to a user's emotions.

The results highlight how a particular neural technology may be used to detect and monitor an individual's emotions over time. The resulting system has potential for use in automated music therapy for individuals with emotion regulation problems. This is highlighted by the case study demonstrating the systems potential with an individual with Huntington's disease.

\section{Case study 2: Driving}

Neural technology for measuring emotions has many other potential applications. In this section we present a second such possible application; the use of neural technology to measure the emotional states of drivers.

Driving is an area of day-to-day life which can be negatively influenced by emotional state (stress, road rage, and so forth). Some recent studies have used bio-physiological measurement to attempt to help quantify drivers' emotional states and to consider whether there are any particular independent variables which might help drivers to be more mindful of their emotional state. The study outlined below considered acoustic cues according to two different types of engine, and aimed to evaluate the link between acoustic environment, driver mood, and bio-physiological response, to the new electric taxi.

Four London taxi drivers participated in an experiment using discrete brain and body sensors to measure bio-physiological cues as they drove an electric and a diesel taxi.

Acoustic cues extracted from a stereo recording suggested that the electric vehicle cab was not necessarily quieter, but had an increased dynamic range and lower spectral centroid, both correlates of fatigue in music listening exercises. Drivers described their own experience in the electric vehicle as happier and less stressful than the diesel vehicle, and heart rate and heart rate variability metrics corroborated these descriptions. Brain responses suggested a degree of emotional interaction, and an unexpected finding: correlates of higher concentration when driving the electric vehicle.

\subsection{Background}

Quentin Willson of Top Gear/Fifth Gear described the experience of electric vehicles as "silent speed. A totally new concept for today's drivers". There are a number of proposed acoustic correlates for mental states, and many people in Britain describe their experience of the environment as excessively noisy. 
Previous driving metrics have suggested that heart rate and skin resistance are most correlated with driver stress (Healey \& Picard, 2005).

The ratio and duration of alpha and beta waves in brain activity has been shown to be correlated to stress (Puglisi-Allegra \& Oliverio, 1990) and used as an analogue to stress in evaluation of driver mental state using virtual reality driving simulations (Benoit et al., 2009; Schier, 2000). However, recent improvements in portability, combined sampling, and durability of analogue to digital conversion allows for real-world testing (Ollander, Godin, Charbonnier, \& Campagne, 2016; Rigas, Goletsis, \& Fotiadis, 2012). This experiment combines acoustic measurement (stereo recording) with bio-physiological and self-report metrics conducted on professional London taxi drivers in two conditions: electric and diesel vehicles, across a series of randomized case series trials.

\subsection{Method}

Experiment information sheets were explained, and copies given to participants before undertaking the tests. Consent forms in accordance with the University of York ethics approval process were signed and collected from all participants.

Four professional taxi drivers undertook 12 trials around the Regents park area in the TX4 (diesel) and TX (electric) taxi cabs. Each trial took approximately 20 minutes.

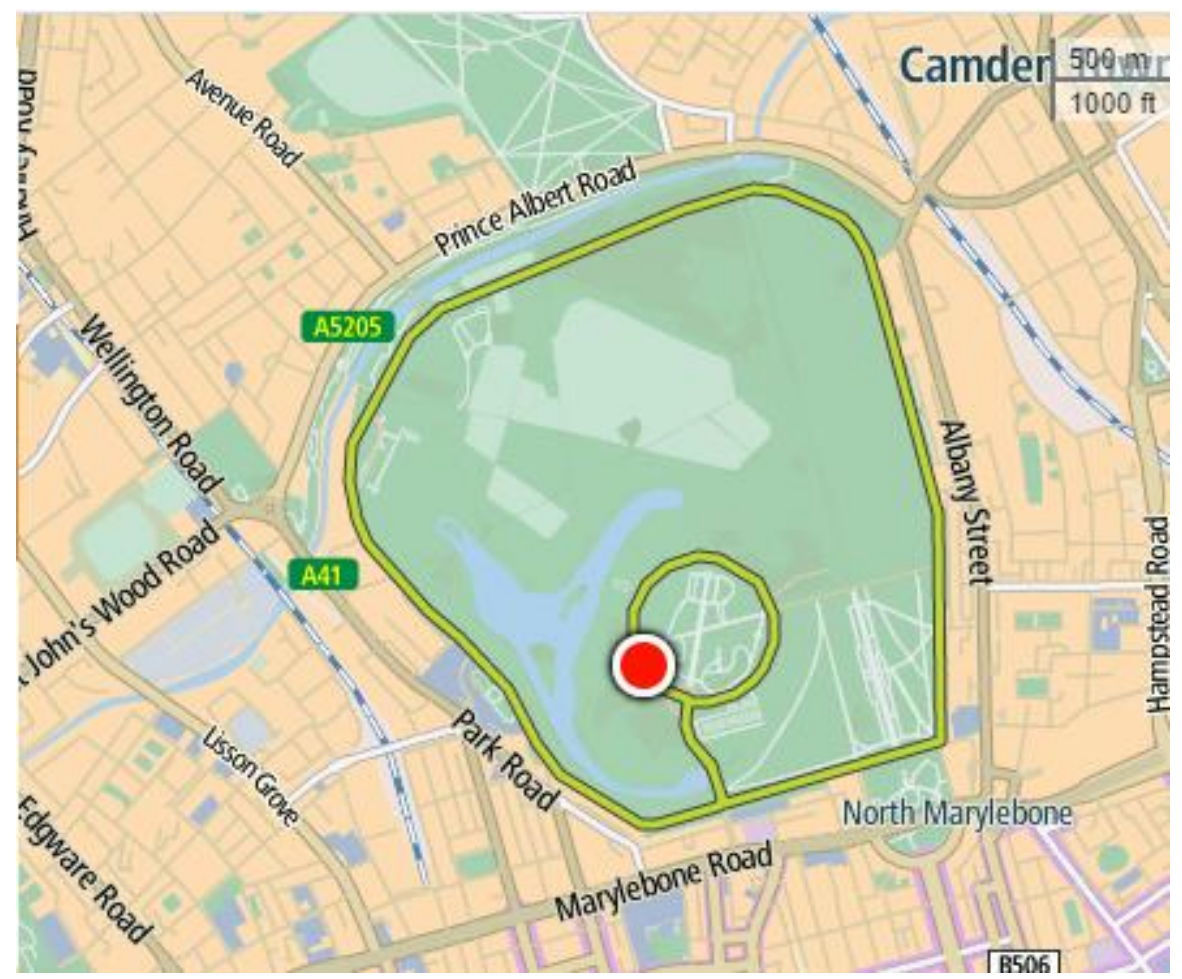

Figure 5. Map of the route taken by the drivers around Regent's Park, London (approx. 3.5 miles).

\subsection{Data collected}


Stereo recording of audio files were taken from the cab at a sampling rate of $44.1 \mathrm{kHz}$, with synchronous $720 \mathrm{p}$ video recording.

Galvanic skin response (electro-dermal activity) was recorded from the left ear lobe of the driver (chosen for safety reasons to allow drivers to look to the right to check their blind spot).

Although galvanic skin response has been previously shown to be useful in evaluating stress, it was difficult to place the sensors required in situ in the accepted position for this experiment. The sensors are traditionally placed over the finger and wrist, which would be impractical for drivers in the real-world data collection scenario. The GSR sensor was therefore placed behind the ear lobe.

The heart rate of each driver was also measured via an optical wrist meter. The wrist meter measured levels of oxy-hemoglobin, heart rate was then inferred from this metric by peak counting.

Electroencephalogram (EEG) was recorded from each driver via dry electrodes with 8 channels placed at positions Fp1, Fp2, C3, C5, P7, P8, O1, and O2 in the 10/20 configuration. The EEG was sampled at $250 \mathrm{~Hz}$ and measured at an impedance of $10 \mathrm{k} \Omega$.

Self-reports were taken from each driver after each trial across 5 descriptors drawn from a survey of psychological evaluation of driving conditions, using a 9-point Likert scale, with 1 being 'least' and 10 being 'most'. The evaluation responses were: stress, anger, distraction, fear, and happiness. Additionally, a 3-channel accelerometer was used to record driver movement, with data recorded at a sampling rate of $250 \mathrm{~Hz}$. The accelerometer was mounted on a small 3D printed plastic frame just above the nape of the neck.

\subsection{Analysis techniques}

Readings for each vehicle type were summed and passed through an acoustic feature analysis pipeline (custom software, MATLAB). The values of five features were calculated: Peaks, RMS, Dynamic range, auto correlation, crest factor, and spectral centroid.

The EEG was low pass filtered at $50 \mathrm{~Hz}$ and then Butterworth filtered in the alpha and beta ranges. EEG was then inverse convolved with the accelerometer data to smooth the data with significant head movement. This approach was inspired by work in (Luu \& Dinh, 2018) and interested readers may also wish to consult (Ian Daly, Billinger, Scherer, \& Mueller-Putz, 2013) for an alternative approach to solving the same problem.

Additionally, heart rate variability was also calculated on a per participant and per trial basis.

Across each self, -report and descriptor per vehicle the mean, standard deviation, and variance of each of the above features were taken. These were compared between both types of vehicle.

\subsection{Results}


Self-report measures are shown in Table 3 and indicate that participants described their experience of driving the electric vehicle as:

1. Less stressful than driving the diesel vehicle.

2. Making them neither more or less angry than driving the diesel vehicle.

3. Less distracting than driving the diesel vehicle.

4. Making them neither more or less afraid than driving the diesel vehicle.

5. Making them happier than driving the diesel vehicle

Table 3. Self-reported responses to the electric and diesel vehicle conditions made by drivers using a 9-point Likert scale (with 1 being 'least' and 10 being 'most') across five descriptors. Collected after each trial. 'Var' denotes the variance of the reports.

\begin{tabular}{llllll} 
Diesel & Stress & Anger & Distraction & Fear & Happiness \\
\hline Mean & 3.50 & 1.33 & 3.83 & 1.17 & 6.83 \\
STD & 0.89 & 0.52 & 1.97 & 0.00 & 1.41 \\
Var. & 0.80 & 0.27 & 3.90 & 0.00 & 2.00 \\
\hline & & & & & \\
Electric & Stress & Anger & Distraction & Fear & Happiness \\
\hline Mean & 2.17 & 1.33 & 2.67 & 1.50 & 8.17 \\
STD & 1.47 & 0.52 & 1.51 & 1.84 & 1.47 \\
Var. & 2.17 & 0.27 & 2.27 & 0.70 & 2.17 \\
\hline
\end{tabular}

Heart rate: Drivers generally exhibited slightly lower mean heart rate in the electric vehicle driving trials, as shown in Figure 6.

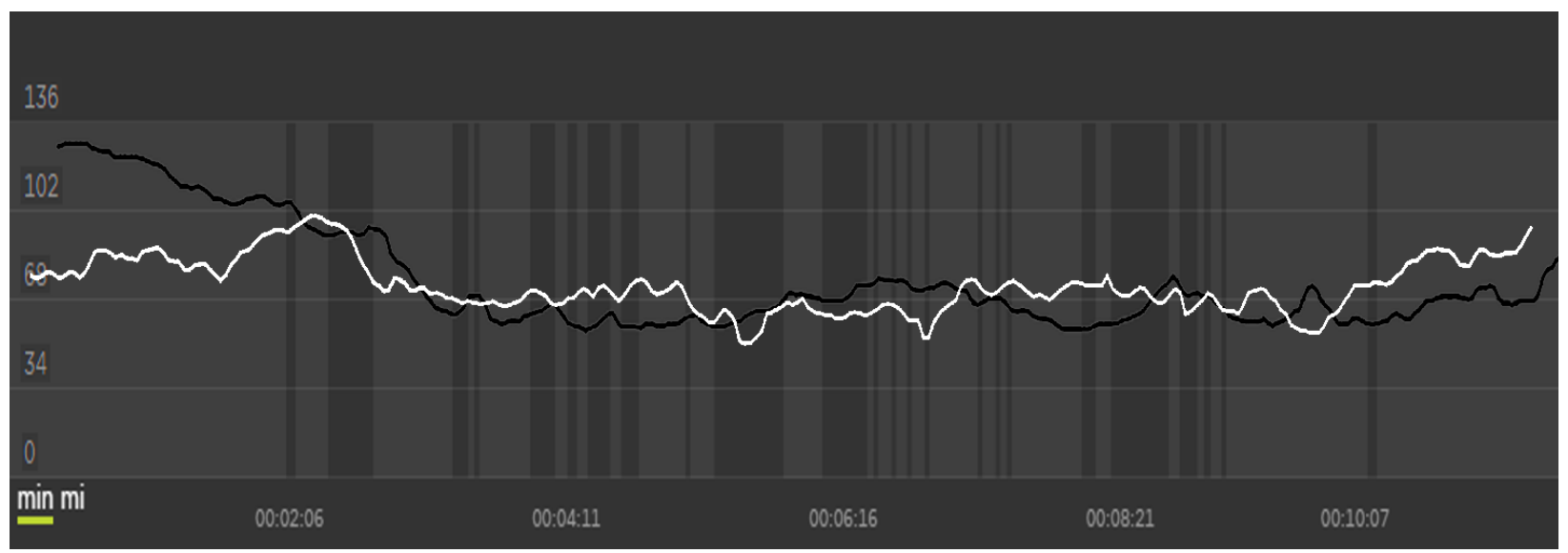

Figure 6. Heart rate across diesel (white line) and electric (black line) care driving trials for driver A.

Note, the overall lower heart rate comparing the electric trial to the diesel trial for the same driver. This pattern can be seen in plots for 3 out of 4 of the driver participants (drivers A, B, and C). However, driver D showed a markedly similar mean heart rate when comparing diesel and electric trials, as shown in Figure 7. 


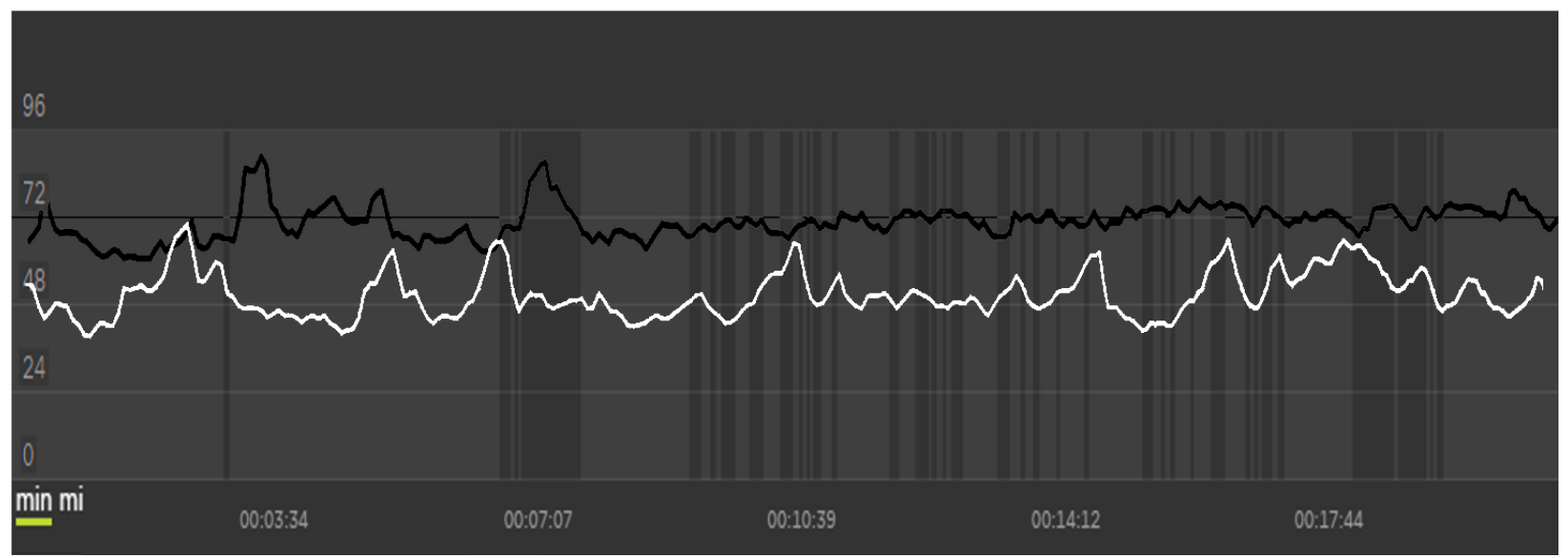

Figure 7. Heart rate across diesel (white) and electric (black) vehicle driving trials for driver D. Note only a slight reduction was observed in the range of the heart rate and increased variability in diesel driving trials, with large peaks and troughs occurring throughout the trial.

Whilst data regarding participant hearing was not collected, it was noted that the participant in question (driver D above) did wear a hearing aid. This suggests that whilst the sound world may have minimal influence on the mean heart rate, there was nevertheless a degree of heart rate variability increase in the diesel trials in comparison to the electric vehicle trials in this case, as was also seen in the other participant trials. All drivers exhibited lower heart rate variability in the electric vehicle driving trials.

The drivers in the diesel trials frequently exhibited frontal asymmetry, which has been correlated with both negative and positive affect (Schaffer, Davidson, \& Saron, 1983). Asymmetry in itself is not necessarily a useful indicator of affect in this case (Chu, Tranel, \& Damasio, 1994) and is to some extent a default, though frontal and parietal asymmetry has been shown more recently in EEG studies to be well correlated with the valence of the emotion (Palmiero \& Piccardi, 2017). Perhaps more unusually, the electric trials exhibit less asymmetry and higher levels of beta frequency band power in comparison to alpha frequency band power. Alpha frequencies tend to be correlated with restful or relaxed states of mind, and beta frequencies with concentration. This finding is thus quite unexpected for the electric trials -- drivers reported more happiness, less stress, in the electric vehicle -- so it may be that the electric vehicle was less distracting and in fact allowed drivers to concentrate more, resulting in less overall fatigue at the end of a driving session.

The GSR showed little difference between either vehicle type across the trials but these values were also below the statistical significance threshold. A distribution is shown in Figure 8. 


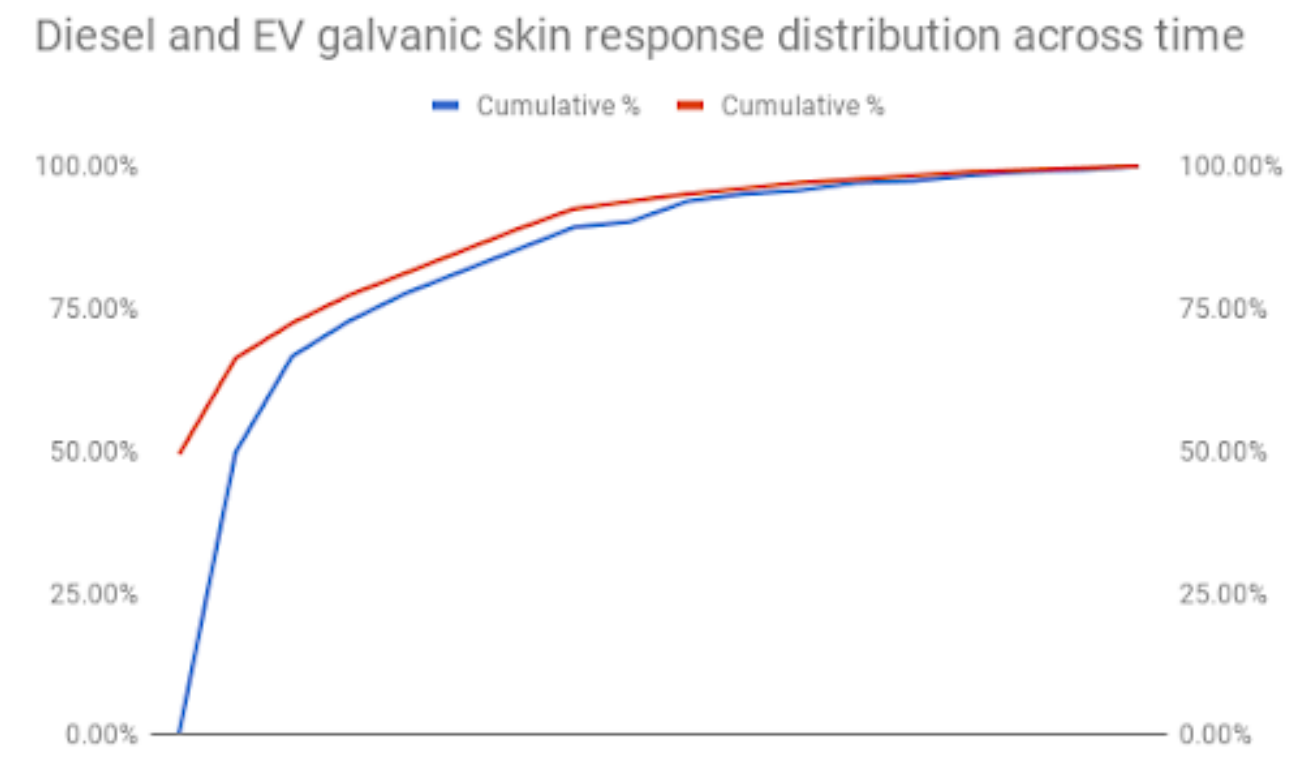

Figure 8. Distribution of galvanic skin response values across timestamp values for recordings (Electric vehicle in blue, Diesel vehicle in red).

Six acoustic features (peak values, root mean square amplitude, dynamic range, auto-correlation, crest factor, and spectral centroid) were extracted using the signal processing toolbox in MATLAB and are shown in Table 4.

Table 4. A comparison of acoustic features extracted from stereo recordings made inside the diesel and electric vehicles during each trial. Although the order of the trials was randomized during the collection, for ease of comparison here they are presented as follows: odd numbers are recordings from the diesel cab (shaded grey), even numbers from the electric cab (no shading).

\begin{tabular}{|l|l|l|l|l|l|}
\hline $\begin{array}{l}\text { Peaks }(\mathrm{max}, \\
\text { min) }\end{array}$ & RMS & $\begin{array}{l}\text { Range } \\
(\mathrm{dBFS})\end{array}$ & Autocorrelation & $\begin{array}{l}\text { Crest factor } \\
(\mathrm{dB})\end{array}$ & $\begin{array}{l}\text { Spectral } \\
\text { centroid }\end{array}$ \\
\hline $\begin{array}{l}\text { Max 0.944 } \\
\text { Min -0.943 }\end{array}$ & 0.140 & 89.811 & 2.802 & 16.560 & 4112.533 \\
\hline $\begin{array}{l}\text { Max 0.723 } \\
\text { Min -0.708 }\end{array}$ & 0.108 & 87.493 & 2.807 & 16.519 & 3290.366 \\
\hline $\begin{array}{l}\text { Max 0.856 } \\
\text { Min -0.877 }\end{array}$ & 0.164 & 88.962 & 10.255 & 14.364 & 6789.525 \\
\hline $\begin{array}{l}\text { Max 0.914 } \\
\text { Min -0.929 }\end{array}$ & 0.172 & 89.528 & 7.383 & 14.485 & 6704.825 \\
\hline $\begin{array}{l}\text { Max 0.997 } \\
\text { Min- 1.000 }\end{array}$ & 0.176 & 90.286 & 4.192 & 15.057 & 6772.741 \\
\hline $\begin{array}{l}\text { Max 0.635 } \\
\text { Min -0.614 }\end{array}$ & 0.113 & 86.367 & 15.710 & 14.974 & 7980.007 \\
\hline $\begin{array}{l}\text { Max 0.909 } \\
\text { Min -0.866 }\end{array}$ & 0.169 & 89.482 & 15.710 & 14.565 & 6750.248 \\
\hline $\begin{array}{l}\text { Max 0.909 } \\
\text { Min -0.866 }\end{array}$ & 0.169 & 89.482 & 15.710 & 14.565 & 6750.241 \\
\hline $\begin{array}{l}\text { Max 0.999 } \\
\text { Min - } 1.000\end{array}$ & 0.276 & 90.309 & 0.145 & 11.186 & 11194.000 \\
\hline
\end{tabular}




\begin{tabular}{|l|l|l|l|l|l|}
\hline $\begin{array}{l}\text { Max 0.916 } \\
\text { Min -0.893 }\end{array}$ & 0.177 & 89.551 & 1.432 & 14.268 & 5684.410 \\
\hline $\begin{array}{l}\text { Max 0.856 } \\
\text { Min -0.877 }\end{array}$ & 0.164 & 88.962 & 10.255 & 14.364 & 6789.525 \\
\hline $\begin{array}{l}\text { Max 0.914 } \\
\text { Min -0.929 }\end{array}$ & 0.172 & 89.528 & 7.383 & 14.485 & 6704.825 \\
\hline
\end{tabular}

The electric vehicle samples indicate lower peak values in comparison to the diesel vehicle recordings, with higher spectral centroid in some samples in the diesel vehicle, and slightly lower mean amplitude (RMS) in the electric vehicle. Surprisingly, some electric vehicle trials indicated a larger dynamic range, though the variance across the dynamic range in all trials was minimal, consistently less than $5 \mathrm{~dB}$ full scale. In layman's terms, these descriptors would typically correlate to a slightly sharper (higher spectral centroid), louder (higher RMS and lower dynamic range) perceived sound in the diesel vehicle. The lower dynamic range might be directly correlated to the idle engine sound of the diesel vehicle as these analyses were extracted from complete trials.

\subsection{Conclusions}

Drivers self-reported little difference in anger or fear between either vehicle type, with no variation in response to anger and minimal variation in response to fear across the two vehicle conditions. Given these are professional drivers with many decades of experience on London roads, it is perhaps unsurprising that the drivers felt little sense of anger or fear undergoing these trials. The electric vehicle was reported to be less stressful and less distracting, as well as a happier driving experience by the drivers than the diesel vehicle - with the largest improvement being in self-reported happiness. The only diesel vehicle ratings with significant inter-participant variation were for the distraction descriptor. There was a greater degree of inter-participant variation amongst the electric vehicle ratings than the diesel ratings for stress, and to a lesser extent, happiness.

The EEG showed correlates in various regions, particularly asymmetry, suggesting different emotional responses in each trial, but more significantly a greater level of beta frequency band power in the electric vehicle trials than in the diesel vehicle trials. Alpha and beta frequencies have well documented and agreed upon correlates in relaxation (alpha), and active concentration (beta). We hypothesized, based on the self-report measures, that the electric vehicle, which drivers described as more fun and less stressful, would be accompanied by higher levels of alpha than beta - but the opposite appears to be the case. This might be because drivers were in fact able to concentrate more without the noise of the diesel engine, which becomes most noticeable when the vehicle is at a standstill (e.g., waiting at lights).

For the majority of trials, there was a marked difference in mean heart rate, though this is not in itself a prominent correlate of mental state. Heart rate variability (HRV) however is a well understood correlate for stressed mental states (Kim, Cheon, Bai, Lee, \& Koo, 2018), and, even in the case of the driver who exhibited little difference in mean heart rate between the two conditions, there was a marked increase in HRV in the diesel vehicle trials. The driver who exhibited no significant difference in mean rate between the two trials did wear a hearing aid 
device -- which suggests there might be a correlation between the sound world and the resulting influence on heart rate -- but this would be impossible to establish without a larger number of participants.

Whilst the biophysiological responses are not necessarily what would be expected given the selfreport results, there seems to be a strong case to suggest that the drivers in the electric vehicle trials were less mentally and physically fatigued by the drive.

\section{Conclusions and future research directions}

Computer systems that are able to measure their users current emotional (affective) states have tremendous potential to provide improvements in therapeutic applications for a wide range of clinical populations, as well as their potential to improve interactions with computer systems and other technologies for general users. Neural technology that is able to provide such a metric, therefore has great potential.

This chapter introduced and discussed some of the most well-known and frequently investigated neural technology-based metrics for measuring emotion. These metrics most frequently make use of the electroencephalogram (EEG), but can also make use of other neuroimaging technologies, such as functional magnetic resonance imaging or functional near infrared spectroscopy. These metrics provide a quantifiable objective measure of an individual's current felt emotion.

Neural technology-based measures of an individual's current emotions have been shown to be able to identify an individual's current level of valence and arousal. Furthermore, when they are coupled to physiological measures, they may provide relatively more accurate measures of a wide range of different emotional states from an individual in a variety of different contexts.

Neural technology-based measures of affect have a large number of potential applications. Emotion is an intrinsic part of the human experience and, increasingly, our experience of the world is mediated through information communications technology. Thus, accurate metrics that allow our technology to understand how we are feeling are increasingly important in building systems that adapt and react optimally to our needs.

However, there are numerous challenges to overcome before neural technology can be most effectively used in a wide range of applications. Specifically, neural technology for measuring emotions faces challenges related to its accuracy, setup time and convenience, and its reliability.

The accuracy of any neural technology-based metrics of emotion is largely mediated by the quality of the signals used, the variability of those signals, the quality of the signal processing and machine learning techniques used to interpret those signals, and the unreliability of the ground-truth measure upon which those methods are calibrated. For example, EEG suffers from artifact contamination from a variety of sources and, thus, constitutes a noisy, non-linear, and non-stationary measure of brain activity (Schomer \& Lopes de Silva, 2011). Furthermore, the machine learning methods trained to interpret this signal are, typically, trained via a supervised learning approach and, the ground truth labels used in this approach can be unreliable as 
individuals may not be able, or willing, to accurately quantify their emotional responses in every situation.

Setup time and convenience of neural technology is also a key impediment to its wide spread adoption. EEG is the one of the most suitable forms of neural technology for measuring emotion due to its relative low cost, relative portability, and high time resolution However, it still takes between 5 to 20 minutes to setup an EEG recording, a process that typically involves mounting electrodes within a cap and forming a conductive bridge with the scalp via conductive gel, a process that can be uncomfortable and inconvenient. Modern EEG systems seek to overcome these issues via the use of "dry electrode" technology, which does away with gel, and wireless systems, that remove the need for attaching multiple wires to the user. However, further advances in setup speed and convenience are still needed.

Furthermore, other physiological measures are not well suited to all situations. An example of this is seen in our driver case study, in which GSR could not be used in the conventional way (recording from the hands) as this would be unsafe in a driving context.

Finally, the reliability of neural technology is hampered by factors including the inherent nonstationarity of brain activity, as well as artifact contamination of the signal. Signal processing techniques seek to overcome these via automated cleaning of the EEG and more accurate and robust classification methods. However, considerable advances are still needed in this area before neural technology can be sufficiently reliable.

Future research in a wide range of fields will benefit from overcoming these problems and from identifying and building neural technology that is able to accurately, reliably, and conveniently identify an individual's current emotional state.

\section{References}

Aftanas, L. I., Varlamov, A. A., Pavlov, S. V., Makhnev, V. P., \& Reva, N. V. (2001). Affective picture processing: event-related synchronization within individually defined human theta band is modulated by valence dimension. Neuroscience Letters, 303(2), 115-118. https://doi.org/10.1016/S0304-3940(01)01703-7

Barrett, L. F., Mesquita, B., Ochsner, K. N., \& Gross, J. J. (2007). The experience of emotion. Annual Review of Psychology, 58, 373-403. https://doi.org/10.1146/annurev.psych.58.110405.085709

Bates, G., Tabrizi, S. J., \& Jones, L. (2014). Huntington's Disease. Oxford: Oxford University Press. Retrieved from https://books.google.co.uk/books?id=i4kAwAAQBAJ\&printsec $=$ frontcover\&dq=huntington $\% 27 \mathrm{~s}+$ disease $\&$ hl $=$ en $\& s a=X \& v e d=0$ ahUKEwih9rHI287RAhUBVxoKHY7TANwQ6AEIKDAC $\# v=$ onepage\&q=huntington's disease $\& \mathrm{f}=$ false

Benoit, A., Bonnaud, L., Caplier, A., Ngo, P., Lawson, L., Trevisan, D. G., ... Chanel, G. (2009). Multimodal focus attention and stress detection and feedback in an augmented driver simulator. Personal and Ubiquitous Computing, 13(1), 33-41. https://doi.org/10.1007/s00779-007-0173-0

Blood, A. J., \& Zatorre, R. J. (2001). Intensely pleasurable responses to music correlate with 
activity in brain regions implicated in reward and emotion. Proceedings of the National Academy of Sciences of the United States of America, 98(20), 11818-11823. https://doi.org/10.1073/pnas.191355898

Bradt, J., Magee, W. L., Dileo, C., Wheeler, B. L., \& McGilloway, E. (2010). Music therapy for acquired brain injury. The Cochrane Database of Systematic Reviews, (7), CD006787. https://doi.org/10.1002/14651858.CD006787.pub2

Canli, T., Desmond, J. E., Zhao, Z., Glover, G., \& Gabrieli, J. D. (1998). Hemispheric asymmetry for emotional stimuli detected with fMRI. Neuroreport, 9(14), 3233-3239. Retrieved from http://www.ncbi.nlm.nih.gov/pubmed/9831457

Chu, C.-C., Tranel, D., \& Damasio, H. (1994). How reliable are occipital asymmetry measurements? Neuropsychologia, 32(12), 1503-1513. https://doi.org/10.1016/00283932(94)90122-8

Coan, J. A., \& Allen, J. J. . (2004a). Frontal EEG asymmetry as a moderator and mediator of emotion. Biological Psychology, 67(1-2), 7-50. https://doi.org/10.1016/j.biopsycho.2004.03.002

Coan, J. A., \& Allen, J. J. . (2004b). Frontal EEG asymmetry as a moderator and mediator of emotion. Biological Psychology, 67(1-2), 7-50. https://doi.org/10.1016/j.biopsycho.2004.03.002

Cowie, R., Douglas-Cowie, E., Savvidou, S., McMahon, E., Sawey, M., \& Schröder, M. (2000). "FEELTRACE": An Instrument For Recording Perceived Emotion In Real Time. In Proceedings of the ISCA Workshop on Speech and Emotion (pp. 19-24). Retrieved from http://citeseerx.ist.psu.edu/viewdoc/summary?doi=10.1.1.58.7528

Daly, I., Billinger, M., Scherer, R., \& Mueller-Putz, G. (2013). On the automated removal of artifacts related to head movement from the EEG. IEEE Transactions on Neural Systems and Rehabilitation Engineering, 21(3), 427-434.

Daly, I., Ho, A., Marcon, J., Hwang, F., Williams, D., Kirke, A., ... Nasuto, S. (2017). Affective Brain Computer Music Interfacing: A Case Study Of Use By An IndividualWith Huntington's Disease. In Proceedings of the Graz Brain-computer interfacing conference 2017.

Daly, I., Malik, A., Hwang, F., Roesch, E., Weaver, J., Kirke, A., ... Nasuto, S. J. (2014). Neural correlates of emotional responses to music: an EEG study. Neuroscience Letters, 573, 5257. https://doi.org/10.1016/j.neulet.2014.05.003

Daly, I., Malik, A., Weaver, J., Hwang, F., Nasuto, S. J., Williams, D., ... Miranda, E. (2015). Identifying music-induced emotions from EEG for use in brain-computer music interfacing. In 6th Affective Computing and Intelligent Interaction.

Daly, I., Williams, D., Hallowell, J., Hwang, F., Kirke, A., Malik, A., ... Nasuto, S. J. (2015). Music-induced emotions can be predicted from a combination of brain activity and acoustic features. Brain and Cognition, 101, 1-11. https://doi.org/10.1016/j.bandc.2015.08.003

Daly, I., Williams, D., Kirke, A., Weaver, J., Malik, A., Hwang, F., ... Nasuto, S. J. (2016a). Affective brain-computer music interfacing. Journal of Neural Engineering, 13(4), 046022. https://doi.org/10.1088/1741-2560/13/4/046022

Daly, I., Williams, D., Kirke, A., Weaver, J., Malik, A., Hwang, F., ... Nasuto, S. J. (2016b). Affective brain-computer music interfacing. Journal of Neural Engineering, 13(4), 046022. https://doi.org/10.1088/1741-2560/13/4/046022

Daly, I., Williams, D., Kirke, A., Weaver, J., Malik, A., Hwang, F., ... Nasuto, S. J. (2016). An Affective Brain-computer music Interface. In Proceedings of the 6th International Brain- 
Computer Interface Meeting, organized by the BCI Society. https://doi.org/10.3217/978-385125-467-9-227

Darwin, C. (1998). The Expression of the Emotions in Man and Animals. Oxford University Press.

Davidson, R. J., Abercrombie, H., Nitschke, J. B., \& Putnam, K. (1999). Regional brain function, emotion and disorders of emotion. Current Opinion in Neurobiology, 9(2), 228-234. https://doi.org/10.1016/S0959-4388(99)80032-4

Eerola, T. (2012). Modeling listeners' emotional response to music. Topics in Cognitive Science, 4(4), 607-624. https://doi.org/10.1111/j.1756-8765.2012.01188.x

Effron, D. A., Niedenthal, P. M., Gil, S., \& Droit-Volet, S. (2006). Embodied temporal perception of emotion. Emotion, 6(1), 1-9. https://doi.org/10.1037/1528-3542.6.1.1

Erkkilä, J., Punkanen, M., Fachner, J., Ala-Ruona, E., Pöntiö, I., Tervaniemi, M., ... Gold, C. (2011). Individual music therapy for depression: randomised controlled trial. The British Journal of Psychiatry: The Journal of Mental Science, 199(2), 132-139. https://doi.org/10.1192/bjp.bp.110.085431

Fazli, S., Mehnert, J., Steinbrink, J., Curio, G., Villringer, A., Müller, K.-R., \& Blankertz, B. (2012). Enhanced performance by a hybrid NIRS-EEG brain computer interface. NeuroImage, 59(1), 519-529. https://doi.org/10.1016/j.neuroimage.2011.07.084

Fingelkurts, A. A., Fingelkurts, A. A., Rytsälä, H., Suominen, K., Isometsä, E., \& Kähkönen, S. (2007). Impaired functional connectivity at EEG alpha and theta frequency bands in major depression. Human Brain Mapping, 28(3), 247-261. https://doi.org/10.1002/hbm.20275

Fontaine, J. R. J., Scherer, K. R., Roesch, E. B., \& Ellsworth, P. C. (2007). The World of Emotions is not Two-Dimensional. Psychological Science, 18(12), 1050-1057. https://doi.org/10.1111/j.1467-9280.2007.02024.x

Healey, J. A., \& Picard, R. W. (2005). Detecting Stress During Real-World Driving Tasks Using Physiological Sensors. IEEE Transactions on Intelligent Transportation Systems, 6(2), 156166. https://doi.org/10.1109/TITS.2005.848368

Heller, W. (1993). Neuropsychological mechanisms of individual differences in emotion, personality, and arousal. Neuropsychology, 7(4), 476-489. https://doi.org/10.1037/08944105.7.4.476

Hou, J., Song, B., Chen, A. C. N., Sun, C., Zhou, J., Zhu, H., \& Beauchaine, T. P. (2017). Review on Neural Correlates of Emotion Regulation and Music: Implications for Emotion Dysregulation. Frontiers in Psychology, 8, 501. https://doi.org/10.3389/fpsyg.2017.00501

Hunter, P. G., \& Schellenberg, E. G. (2010). Music and Emotion. In Music Perception, Springer Handbook of Auditory Research (pp. 129-164).

Irene Winkler, Mark Jäger, Vojkan Mihajlović, T. T. (2010). Frontal EEG Asymmetry Based Classification of Emotional Valence using Common Spatial Patterns. World Academy of Science, Engineering and Technology, 69. Retrieved from http://citeseerx.ist.psu.edu/viewdoc/summary?doi=10.1.1.190.6511

Ishii, R., Canuet, L., Ishihara, T., Aoki, Y., Ikeda, S., Hata, M., ... Takeda, M. (2014). Frontal midline theta rhythm and gamma power changes during focused attention on mental calculation: an MEG beamformer analysis. Frontiers in Human Neuroscience, 8, 406. https://doi.org/10.3389/fnhum.2014.00406

Jones, N. A., \& Fox, N. A. (1992). Electroencephalogram asymmetry during emotionally evocative films and its relation to positive and negative affectivity. Brain and Cognition, 20(2), 280-299. Retrieved from http://www.ncbi.nlm.nih.gov/pubmed/1449758 
Kassam, K. S., Markey, A. R., Cherkassky, V. L., Loewenstein, G., \& Just, M. A. (2013). Identifying Emotions on the Basis of Neural Activation. PLoS ONE, 8(6), e66032. https://doi.org/10.1371/journal.pone.0066032

Kim, H.-G., Cheon, E.-J., Bai, D.-S., Lee, Y. H., \& Koo, B.-H. (2018). Stress and Heart Rate Variability: A Meta-Analysis and Review of the Literature. Psychiatry Investigation, 15(3), 235-245. https://doi.org/10.30773/pi.2017.08.17

Koelsch, S. (2010). Towards a neural basis of music-evoked emotions. Trends in Cognitive Sciences, 14(3), 131-137. https://doi.org/10.1016/j.tics.2010.01.002

Koelsch, S. (2014). Brain correlates of music-evoked emotions. Nature Reviews Neuroscience, 15(3), 170-180. https://doi.org/10.1038/nrn3666

Kreibig, S. D. (2010). Autonomic nervous system activity in emotion: A review. Biological Psychology, 84(3), 394-421. https://doi.org/10.1016/j.biopsycho.2010.03.010

Lee, Y.-Y., \& Hsieh, S. (2014). Classifying Different Emotional States by Means of EEG-Based Functional Connectivity Patterns. PLoS ONE, 9(4), e95415. https://doi.org/10.1371/journal.pone.0095415

Livingstone, R. S., \& Thompson, W. F. (2009). The emergence of music from the theory of mind. Musicae Scientiae, 13(2 Suppl), 83-115. https://doi.org/10.1177/1029864909013002061

Luu, L., \& Dinh, A. (2018). Artifact Noise Removal Techniques on Seismocardiogram Using Two Tri-Axial Accelerometers. Sensors, 18(4), 1067. https://doi.org/10.3390/s18041067

Mehrabian, A. (1996). Pleasure-arousal-dominance: A general framework for describing and measuring individual differences in Temperament. Current Psychology, 14(4), 261-292. https://doi.org/10.1007/BF02686918

Mier, D., Lis, S., Neuthe, K., Sauer, C., Esslinger, C., Gallhofer, B., \& Kirsch, P. (2010). The involvement of emotion recognition in affective theory of mind. Psychophysiology, 47(6), no-no. https://doi.org/10.1111/j.1469-8986.2010.01031.x

Müller-Putz, G. R., Breitwieser, C., Cincotti, F., Leeb, R., Schreuder, M., Leotta, F., \& Et.al. (2011). Tools for Brain-Computer Interaction: A General Concept for a Hybrid BCI. Front Neuroinform, 5, 30. https://doi.org/10.3389/fninf.2011.00030

Nicolaou, N., Malik, A., Daly, I., Weaver, J., Hwang, F., Kirke, A., ... Nasuto, S. J. (2017). Directed Motor-Auditory EEG Connectivity Is Modulated by Music Tempo. Frontiers in Human Neuroscience, 11, 502. https://doi.org/10.3389/fnhum.2017.00502

Nicolas-Alonso, L. F., \& Gomez-Gil, J. (2012). Brain computer interfaces, a review. Sensors (Basel, Switzerland), 12(2), 1211-1279. https://doi.org/10.3390/s120201211

Niedermeyer, E., \& Silva, F. H. L. Da. (2005). Electroencephalography: basic principles, clinical applications, and related fields. Lippincott Williams \& Wilkins.

Ollander, S., Godin, C., Charbonnier, S., \& Campagne, A. (2016). Selection of the most relevant physiological features for classifying emotion. In 3rd International Conference on Physiological Computing Systems (PhyCS 2016). Retrieved from https://hal.archivesouvertes.fr/hal-01378328

Ortony, A., Clore, G. L., \& Collins, A. (1988). The Cognitive Structure of Emotions. Cambridge: Cambridge University Press. https://doi.org/10.1017/CBO9780511571299

Palmiero, M., \& Piccardi, L. (2017). Frontal EEG Asymmetry of Mood: A Mini-Review. Frontiers in Behavioral Neuroscience, 11, 224. https://doi.org/10.3389/fnbeh.2017.00224

Pfurtscheller, G., Allison, B. Z., Brunner, C., Bauernfeind, G., Solis-Escalante, T., Scherer, R., ... Birbaumer, N. (2010). The hybrid BCI. Frontiers in Neuroprosthetics, 4(30). 
Pridmore, S. A. (1990). The prevalence of Huntington's disease in Tasmania. The Medical Journal of Australia, 153(3), 133-134. Retrieved from http://www.ncbi.nlm.nih.gov/pubmed/2142982

Puglisi-Allegra, S., \& Oliverio, A. (1990). Psychobiology of Stress. Springer Netherlands.

Raichle, M. E. (2011). The restless brain. Brain Connectivity, 1(1), 3-12. https://doi.org/10.1089/brain.2011.0019

Reeve, J. (2014). Understanding Motivation and Emotion (Vol. 3). John Wiley \& Sons. Retrieved from https://books.google.com/books?hl=en\&lr=\&id=zfOyBwAAQBAJ\&pgis=1

Rigas, G., Goletsis, Y., \& Fotiadis, D. I. (2012). Real-Time Driver's Stress Event Detection. IEEE Transactions on Intelligent Transportation Systems, 13(1), 221-234. https://doi.org/10.1109/TITS.2011.2168215

Rogenmoser, L., Zollinger, N., Elmer, S., \& Jäncke, L. (2016). Independent component processes underlying emotions during natural music listening. Social Cognitive and Affective Neuroscience, 11(9), 1428-1439. https://doi.org/10.1093/scan/nsw048

Russell, J. A. (1980). A circumplex model of affect. Journal of Personality and Social Psychology, 39(6), 1161-1178.

Sacks, O. (2006). The power of music. Brain : A Journal of Neurology, 129(Pt 10), 2528-2532. https://doi.org/10.1093/brain/awl234

Sammler, D., Grigutsch, M., Fritz, T., \& Koelsch, S. (2007). Music and emotion: Electrophysiological correlates of the processing of pleasant and unpleasant music. Psychophysiology, 44(2), 293-304. https://doi.org/10.1111/j.1469-8986.2007.00497.x

Schaffer, C. E., Davidson, R. J., \& Saron, C. (1983). Frontal and parietal electroencephalogram asymmetry in depressed and nondepressed subjects. Biological Psychiatry, 18(7), 753-762. Retrieved from http://www.ncbi.nlm.nih.gov/pubmed/6615936

Schier, M. A. (2000). Changes in EEG alpha power during simulated driving: a demonstration. International Journal of Psychophysiology : Official Journal of the International Organization of Psychophysiology, 37(2), 155-162. Retrieved from http://www.ncbi.nlm.nih.gov/pubmed/10832002

Schimmack, U., \& Grob, A. (2000). Dimensional models of core affect: a quantitative comparison by means of structural equation modeling. European Journal of Personality, 14(4), 21.

Schmidt, L. A., \& Trainor, L. J. (2001). Frontal brain electrical activity (EEG) distinguishes valence and intensity of musical emotions. Cognition \& Emotion, 15(4), 487-500. https://doi.org/10.1080/02699930126048

Schomer, L., \& Lopes de Silva, F. (Eds.). (2011). Niedermeyer's electroencephalography: Basic principles, clinical applications, and related fields (6th ed.). Lippincott Williams \& Wilkins.

Silberman, E. K., \& Weingartner, H. (1986). Hemispheric lateralization of functions related to emotion. Brain and Cognition, 5(3), 322-353. https://doi.org/10.1016/0278-2626(86)900357

Skouras, S., Gray, M., Critchley, H., \& Koelsch, S. (2013). FMRI scanner noise interaction with affective neural processes. PloS One, 8(11), e80564. https://doi.org/10.1371/journal.pone.0080564

Sporns, O. (2007). Brain Connectivity. Scholarpedia, 2(10), 4695. Retrieved from http://www.scholarpedia.org/article/Brain_connectivity

Stikic, M., Johnson, R. R., Tan, V., \& Berka, C. (2014). EEG-based classification of positive and 
negative affective states. Brain-Computer Interfaces, 1(2), 99-112.

https://doi.org/10.1080/2326263X.2014.912883

Vuoskoski, J. K., \& Eerola, T. (2011). Measuring music-induced emotion: A comparison of emotion models, personality biases, and intensity of experiences. Musicae Scientiae, 15(2), 159-173. https://doi.org/10.1177/1029864911403367

Webber, R., Aha, D. W., Muñoz-Ávila, H., \& Breslow, L. A. (2000). Advances in Case-Based Reasoning. Advances in Case-Based Reasoning, 1898, 322-334. https://doi.org/10.1007/3540-44527-7

Williams, D., Kirke, A., Miranda, E., Daly, I., Hwang, F., Weaver, J., \& Nasuto, S. (2017). Affective Calibration of Musical Feature Sets in an Emotionally Intelligent Music Composition System. ACM Transactions on Applied Perception, 14(3), 1-13. https://doi.org/10.1145/3059005

Williams, D., Nasuto, S., Kirke, A., Miranda, E., Daly, I., Hallowell, J., ... Hwang, F. (2015). Investigating Perceived Emotional Correlates of Rhythmic Density in Algorithmic Music Composition. ACM Transactions on Applied Perception, 12(3), 1-21. https://doi.org/10.1145/2749466

Zentner, M., Grandjean, D., \& Scherer, K. R. (2008). Emotions evoked by the sound of music: Characterization, classification, and measurement. Emotion, 8(4), 494-521. https://doi.org/10.1037/1528-3542.8.4.494 